\title{
Two-Year Complete Remission of Diffuse Large B-Cell Lymphoma in an Immunological Nonresponder HIV-Infected Patient: Case Report
}

\author{
Miodrag Vrbic ${ }^{a} \quad$ Ivan Petkovic $^{b} \quad$ Svetislav Vrbic $^{b} \quad$ Maja Jovanovic $^{a}$ \\ Aleksandar Rankovic ${ }^{a} \quad$ Lidija Popovic-Dragonjic $^{a}$ \\ Marina Djordjevic-Spasic ${ }^{a}$ \\ ${ }^{a}$ Clinic of Infectious Diseases, Clinical Centre Nis, Nis, Republic of Serbia; \\ ${ }^{b}$ Clinic of Oncology, Clinical Centre Nis, Nis, Republic of Serbia
}

\section{Keywords}

Diffuse large B-cell lymphoma · HIV infection · Immunological nonresponder

\begin{abstract}
Introduction: HIV-infected patients are affected significantly more frequently by all types of lymphoma, with diffuse large B-cell lymphoma (DLBCL) as the most prevalent histological type. Since the introduction of combination antiretroviral therapy (CART) morbidity and mortality of DLBCL has been markedly reduced, which is primarily interpreted as a result of the drug-mediated immune reconstitution. Case Report: We present a previously healthy, 44year-old HIV-infected man with DLBCL of the oral cavity, treated with immunochemotherapy and CART. During HIV-directed treatment, despite the successful virologic response, a satisfactory immunological response was not achieved. However, the patient had a 2-year complete remission after first-line treatment of DLBCL. Conclusion: Response to CART strongly predicts outcome in patients with DLBCL. Close monitoring of HIV-directed therapy efficacy, especially as to achievement of successful virologic response, independently associated with prolonged survival, is essential for estimating future DLBCL treatment strategies.
\end{abstract}


 Oncology}

\section{Introduction}

In HIV-infected patients, the incidence of non-Hodgkin's lymphoma has been between 60- and 200-times greater compared to that in the general population, and has been responsible for the $16 \%$ of all deaths attributable to AIDS. Diffuse large B-cell lymphoma (DLBCL) is the most prevalent histological type and accounts for up to $80 \%$ of cases [1]. Since the introduction of combination antiretroviral therapy (cART), morbidity and mortality of DLBCL has been reduced, with survival rates approaching those observed in non-HIV patients [2].

The beneficial effects of cART are interpreted as the result of a drug-mediated immune reconstitution and decline of HIV viremia, with the assumption that immune suppression rather than HIV itself is implicated in the pathogenesis of this malignancy [1]. There is a clear correlation between the degree of immunosuppression and the risk of developing DLBCL, with the established fact that a low CD4 count represents an independent and significant predictor of death [3]. Furthermore, in the literature, there are sporadic reports of spontaneous regression of DLBCL following cART therapy alone [4].

On the other hand, in some HIV-infected individuals, the so-called immunological nonresponders, a significant CD4-cell increase cannot be achieved despite prolonged viral suppression with cART, and consequences of this condition are not completely understood [5].

We here present the case of an HIV-positive immunological nonresponder patient with complete remission of DLBCL of the oral cavity.

\section{Case Report}

A previously healthy 44-year-old man presented with a painful, erythematous tumefaction on the soft palate and anterior palatal arch (about $4 \mathrm{~cm}$ in diameter), with a superficial ulceration (about $1.5 \mathrm{~cm}$ in diameter). The lesion had been growing for the past 4 months, despite the outpatient treatment, mainly with antibiotics.

After incisional biopsy of the change, a DLBCL was diagnosed with the following immunohistochemical status: CD3-, CD5-, CD20+, CD10+, MUM1+, Bcl6+, Bcl2-, Ki67+ in 90\% of the cells. With further examination (abiding by the recommendations of the American Cancer Society) the disease was determined to be clinical stage IE, A. The Age-Adjusted International Prognostic Index (aaIPI) score was 0. Bone marrow finding matched the pattern of viral activation.

The serological tests were as follows: HBsAg negative, anti-HCV negative, anti-EBV negative, and anti-HIV positive. HIV infection was confirmed with HIV-1 RNA RT-PCR, with a viral load (VL) of 17,800 copies/mL. CD4 count was 61 cells $/ \mathrm{mm}^{3}$.

The patient was treated with immunochemotherapy, using rituximab plus cyclophosphamide, doxorubicin, vincristine, and prednisone (R-CHOP), in 6 cycles of 21 days. A complete clinical remission of the lesion was achieved after the 5th cycle of therapy. After the 4th and 5th cycle, the patient was treated with short-term granulocyte-colony stimulating factor (G-CSF) therapy due to neutropenia. Simultaneously with immunochemotherapy, cART treatment was administered, with lamivudine plus abacavir, and efavirenz.

During the 2 years of posttreatment follow-up, the patient was regularly restaged every 3 months in the first year, and every 6 months in the second year. So far, the patient has achieved event-free survival, without any detectable foci of pathological fluorodeoxyglucose (FDG) accumulation on positron emission tomography (PET)-CT, performed at the last control visit. 
Regarding the efficacy of cART, VL was measured at 8 weeks, until the achievement of a virologic success (HIV VL $<50$ copies/mL) at the 16th week of therapy, and every 6 months afterwards. During follow-up at 6-month intervals, the initial number of $61 \mathrm{CD} 4$ cells $/ \mathrm{mm}^{3}$ rose to 81 cells $/ \mathrm{mm}^{3}, 118$ cells $/ \mathrm{mm}^{3}, 144$ cells $/ \mathrm{mm}^{3}$, and 174 cells $/ \mathrm{mm}^{3}$, respectively, till the end of the 2nd year of treatment.

PCP and toxoplasmosis were prevented by daily intake of trimethoprim/sulfamethoxazole. AIDS-related conditions and other signs or symptoms were absent.

\section{Discussion}

Non-Hodgkin's lymphomas, in the first place DLBCL, are the third most common group of malignant lesions in the oral cavity, and palatal mucosa is one of the predilection sites, with the typical clinical appearance of a rapidly enlarging local mass, often with superficial ulceration. However, primary DLBCL of the oral cavity is rare, with the prevalence of $3-5 \%$, but they have been increasingly found in HIV-positive patients [6].

Similar to HIV-negative patients, the category of HIV-associated DLBCL is a histopathologically and clinically heterogeneous group. In the presented patient, germinal center Bcell-like (GCB) DLBCL aberrant molecular phenotype, with MUM1 and BCL6 co-expression, as well as a high expression of Ki-67, was identified immunohistochemically. There are numerous, often contradictory interpretations of the clinical significance of MUM1, BCL6, and Ki-67 expression, even more controversial in the HIV-related DLBCL [7].

Therefore, in general, prognostically, the reliable predictor of the possible outcome of DLBCL is still IPI, namely its adaptations aaIPI and revised-IPI. However, relative to the general population, the risk of DLBCL and its worse prognosis remain markedly elevated in HIVpositive patients, even today, in the era of cART and immunochemotherapy. Moreover, considering the fact that overall incidence reduction was not as impressive as with other opportunistic malignancies and infections, lymphoma is now one of the most common causes of AIDS-related deaths [8].

Examining specific risk factors in the HIV-positive setting, it has been found that the number of CD4 cells provides further independent and prognostically significant information [3]. Taking into account that the status of HIV infection has got an important prognostic value, a HIV score has been developed (composed of prior history of AIDS, baseline CD4 count, and VL), capable of predicting overall survival more precisely. Further, by combining the HIV score with known prognostic patient and lymphoma factors, an AIDS-related lymphoma IPI (ARL-IPI) was created, which was significantly better in predicting the risk of death [9].

On the other hand, the impact of all the above-mentioned prognostic factors, measured at the time of DLBCL diagnosis, was reduced with a strong association of efficacy of HIVdirected therapy with prolonged survival [10]. Therefore, the patients achieving undetectable VL had statistically significant superior 2-year overall survival compared to those who do not [11], while it is assumed that a favorable immune reconstitution reduces the risk of serious intercurrent infectious complications and allows the use of curative antilymphoma therapy for the majority of patients.

However, despite the successful virologic response, some patients, the so-called immunological nonresponders, are unable to achieve a satisfactory immune response. The exact background and relevance of this discordant response is not yet clear, and current guide- 
lines do not recommend switching an otherwise suppressive cART regimen. There are multiple definitions of an immunological failure, and they were all fulfilled in the presented case.

With regard to the multifactorial pathogenesis of HIV-related DLBCL, the predominant contribution is believed to be through indirect mechanisms, namely immunosuppression and chronic inflammation. In the absence of coinfection with EBV and HCV as recognized non-Hodgkins' lymphoma carcinogens, such as in the presented case, HIV infection itself creates an environment in which continuous and dysregulated immune activation, in particular permanent antigen-dependent B-cell stimulation, can lead to the emergence of monoclonal B cells $[12,13]$.

That the immune activation, rather than immune deficiency, is the key factor in the pathogenesis of DLBCL is also suggested by a START study, one of the pivotal ART studies, the results of which indicate that immediate initiation of treatment, irrespective of CD4-cell count, significantly reduce, among other events, incidence of HIV-related lymphomas and non-AIDS-defining cancers [14].

As far as the presented case of DLBCL, an immunological nonresponder HIV-infected patient, is concerned, it still remains to determine the significance of 2-year disease-free posttreatment survival as the proposed surrogate end point in clinical trials and milestone of favorable prognosis of DLBCL in the general population [15].

\section{Conclusion}

Despite the reduced incidence and mortality, DLBCL are now one of the most frequent causes of death in HIV-positive patients. The response to cART strongly predicts outcome in patients with DLBCL. Close monitoring of its efficacy, especially the achievement of successful virological response (as the primary goal of HIV-directed therapy), was independently associated with prolonged survival and is necessary for the evaluation of future DLBCL treatment strategies.

\section{Statement of Ethics}

The authors have no ethical conflicts to disclose.

\section{Disclosure Statement}

The authors declare no conflict of interest.

\section{References}

1 Lee B, Bower M, Newsom TD, Nelson M: HIV-related lymphoma. HIV Ther 2010;4:649-659.

2 Navarro JT, Lloveras N, Ribera JM, Oriol A, Mate JL, Feliu E: The prognosis of HIV-infected patients with diffuse large B-cell lymphoma treated with chemotherapy and highly active antiretroviral therapy is similar to that of HIV-negative patients receiving chemotherapy. Haematologica 2005;90:704-706.

-3 Bower M, Gazzard B, Mandalia S: A prognostic index for systemic AIDS-related non-Hodgkin lymphoma treated in the era of highly active antiretroviral therapy. Ann Intern Med 2005;143:265-273.

-4Villafane MF, Corti M: Primary cutaneous B-cell lymphoma successfully treated with highly active antiretroviral therapy alone: a case report and review of the literature. Indian J Dermatol 2011;56:418420 . 
-5 Kelley CF, Kitchen CM, Hunt PW, et al: Incomplete peripheral CD4+ cell count restoration in HIVinfected patients receiving long-term antiretroviral treatment. Clin Infect Dis 2009;48:787-794.

-6 Silva TD, Ferreira CB, Leite GB, de Menezes Pontes JR, Antunes HS: Oral manifestations of lymphoma: a systematic review. Ecancermedicalscience 2016;10:665.

7 Caserman E, Chadburn A: Pathology; in Hentrich M, Barta SK (eds): HIV-Associated Hematological Malignancies. Springer International Publishing, Switzerland, 2016, pp 4-5.

8 Hoffmann C: Malignant lymphomas; in Hoffmann C, Rockstroh J (eds): HIV 2015/2016. Medizin Focus Verlag, Hamburg, 2015, pp 420-452.

-9 Barta SK, Xue X, Wang D, et al: A new prognostic score for AIDS-related lymphomas in the rituximabera. Haematologica 2014;99:1731-1737.

10 Hoffmann C, Wolf E, Fätkenheuer G, et al: Response to highly active antiretroviral therapy strongly predicts outcome in patients with AIDS-related lymphoma. AIDS 2003;17:1521-1529.

11 Antinori A, Cingolani A, Alba L, et al: Better response to chemotherapy and prolonged survival in AIDSrelated lymphomas responding to highly active antiretroviral therapy. AIDS 2001;15:1483-1491.

12 Pelicci PG, Knowles DM 2nd, Arlin ZA, Wieczorek R, Luciw P, Dina D, Basilico C, Dalla-Favera R: Multiple monoclonal B cell expansions and c-myc oncogene rearrangements in acquired immune deficiency syndrome-related lymphoproliferative disorders. Implications for lymphomagenesis. J Exp Med 2003;164:2049-2060.

13 Achenbach CJ, Buchanan AL, Cole SR, et al: HIV viremia and incidence of non-Hodgkin lymphoma in patients successfully treated with antiretroviral therapy. Clin Infect Dis 2014;58:1599-1606.

14 The INSIGHT START Study Group: Initiation of antiretroviral therapy in early asymptomatic HIV infection. N Engl J Med 2015;373:795-807.

15 Jakobsen LH, Bogsted M, de Nully Brown P, et al: Minimal loss of lifetime for patients with diffuse large B-cell lymphoma in remission and event free 24 months after treatment: a Danish population-based study. J Clin Oncol 2017;35:778-784. 